\title{
The novel prognostic marker, EHMT2, is involved in cell proliferation via HSPD1 regulation in breast cancer
}

\author{
SEON-KYU KIM $^{1 *}$, KWANGHO KIM $^{1 *}$, JEA-WOON RYU $^{1 *}$, TAE-YOUNG RYU ${ }^{1}$, \\ JUNG HWA LIM $^{1}$, JUNG-HWA OH ${ }^{2}$, JEONG-KI MIN ${ }^{1,3}$, CHO-ROK JUNG ${ }^{1,3}$, \\ RYUJI HAMAMOTO ${ }^{4}$, MI-YOUNG SON ${ }^{1,3}$, DAE-SOO KIM ${ }^{1,3}$ and HYUN-SOO CHO ${ }^{1,3}$ \\ ${ }^{1}$ Korea Research Institute of Bioscience and Biotechnology, Daejeon 305-333; \\ ${ }^{2}$ Korea Institute of Toxicology (KIT), Daejeon 34114; ${ }^{3}$ Department of Functional Genomics, \\ Korea University of Science and Technology, Daejeon 305-333, Republic of Korea; \\ ${ }^{4}$ Division of Molecular Modification and Cancer Biology, National Cancer Center, Tokyo 104-0045, Japan
}

Received July 23, 2018; Accepted October 16, 2018

DOI: 10.3892/ijo.2018.4608

\begin{abstract}
Molecular classifications of breast cancer (BRC), such as human epidermal growth factor receptor 2 (HER2), luminal $\mathrm{A}$ and luminal $\mathrm{B}$, have been developed to reduce unnecessary treatment by dividing patients with BRC into low- and high-risk progression groups. However, these methods do not cover all of the pathological characteristics of BRC, and investigations into novel prognostic/therapeutic markers are thus continually required. In this study, we identified the overexpression of the histone methyltransferase, euchromatic histone-lysine N-methyltransferase 2 (EHMT2) in BRC samples $(n=1,222)$ and normal samples $(n=113)$ derived from the TCGA portal by performing a BRC tissue microarray. EHMT2 overexpression was clearly associated with a poor prognosis in multiple cohorts of patients with BRC (total, $\mathrm{n}=1,644)$. Furthermore, the knockdown of EHMT2 expression affected cell apoptosis via the downregulation
\end{abstract}

Correspondence to: Dr Hyun-Soo Cho or Dr Mi-Young Son, Korea Research Institute of Bioscience and Biotechnology, 125 Gwahak-ro, Yuseong-gu, Daejeon 305-333, Republic of Korea

E-mail: chohs@kribb.re.kr

E-mail: myson@kribb.re.kr

${ }^{*}$ Co-first authorship

Abbreviations: BRC, breast cancer; H3K9, histone $\mathrm{H} 3$ lysine 9; TCGA, The Cancer Genome Atlas; FBS, fetal bovine serum; DAPI, 4',6'-diamidino-2-phenylindole dihydrochloride; IPA, Ingenuity Pathway Analysis; siCont, siRNA Control; DEGs, differentially expressed genes; GO term, Gene Ontology term; ER, estrogen receptor; PR, progesterone receptor; EHC, EHMT2-high cluster; ELC, EHMT2-low cluster; TNBC, triple-negative breast cancer; LumA, luminal A

Key words: breast cancer, euchromatic histone-lysine $\mathrm{N}$-methyltransferase 2, prognostic value, heat shock protein family $\mathrm{D}$ (Hsp60) member 1, correlations and re-localization of heat shock protein family D (Hsp60) member 1 (HSPD1). In addition, a statistically significant positive correlation between EHMT2 and HSPD1 expression was revealed in the clinical cohorts. On the whole, the findings of this study may assist the development of novel therapeutic strategies and provide a prognostic marker (EHMT2) for patients with BRC.

\section{Introduction}

Breast cancer (BRC) is the leading type of cancer affecting women and can be divided into 5 subtypes as follows: Human epidermal growth factor receptor 2 (HER2), luminal A, luminal B, basal-like and normal-like $(1,2)$. Based on DNA copy number and genome-wide analyses, patients with BRC can be separated into low- and high-risk disease progression groups to avoid unnecessary treatment (3). However, these classification systems do not cover all the pathological heterogeneities of BRC. Thus, the development of more effective diagnostic strategies and novel therapeutic markers is continually required. Among several markers, epigenetic alterations (histone modifications, DNA methylation/demethylation and miRNA regulation) in BRC are recognized as important pathways for BRC proliferation and metastasis $(4,5)$.

The histone methyltransferase, euchromatic histone-lysine N-methyltransferase 2 (EHMT2) mono- and di-methylates lysine 9 on histone $\mathrm{H} 3$ to form heterochromatic regions, leading to gene suppression in cancer (6). In BRC, EHMT2 regulates $\mathrm{BRC}$ metastasis via MSK1 regulation (7) and is involved in $\mathrm{BRC}$ proliferation via the regulation of ferroxidase hephaestin (8), nuclear factor (NF)- $\mathrm{B}(9)$ and Sox 2 protein stability (10). In addition, EHMT2 is associated with a poor prognosis in esophageal squamous cell carcinoma and melanoma $(11,12)$. However, the prognostic characteristics and molecular functions of EHMT2 in BRC are not yet fully understood.

Heat shock protein family D (Hsp60) member 1 (HSPD1), drives T-cell and B-cell activation and positively regulates the production of a number of interferons and interleukins. In cancer, HSPD1 is involved in mitochondrial dysfunction 
and the downregulation of HSPD1 induces cancer cell apoptosis $(13,14)$.Furthermore, several heat shock proteins, such as HSP60, HSP70 and HSP90, have prognostic significance in several types of cancer (15). Thus, the regulation of HSPD1 in BRC is an attractive therapeutic target for BRC treatment.

In this study, we gathered RNA-seq data from BRC tissues $(n=1,222)$ and normal tissues $(n=113)$ derived from The Cancer Genome Atlas (TCGA) and identified EHMT2 overexpression and its prognostic value using multiple patient cohorts $(n=1,644)$. Furthermore, phosphor array and immunocytochemical analyses revealed that alterations in HSPD1 expression levels and cell localization due to EHMT2 knockdown induced cell apoptosis. Moreover, in multiple patient cohorts, a positive association between EHMT2 and HSPDI was observed, suggesting that EHMT2 may be a novel therapeutic and prognostic marker for BRC treatment.

\section{Materials and methods}

Cell culture and reagents. The human BRC cell lines, MCF7 and MDA-MB-231, were cultured in DMEM supplemented with $10 \%$ fetal bovine serum (FBS) and $1 \%$ penicillin/streptomycin in a humidified $5 \% \mathrm{CO}_{2}$ atmosphere at $37^{\circ} \mathrm{C}$ according to the manufacturer's instructions (16). BIX01294 (an EHMT2 inhibitor) was purchased from Abcam (ab141407; Cambridge, MA, USA). The MDA-MB-231 cells were treated with $9 \mu \mathrm{M}$ BIX01294 for $24 \mathrm{~h}$. DMSO was used for the control treatments.

Public datasets of BRC patients, including TCGA data. In total, 3 cohorts $(n=1,644)$ of patients with BRC, including the TCGA data, were used in this study. mRNA expression (RNA-seq) data were obtained from the cBioPortal website ( $\mathrm{n}=817$, TCGA cohort, http://www.cbioportal.org). We downloaded mRNA quantification data (RSEM format), to which $\log 2$ transformation and quantile normalization were applied. For comparative analysis in expression of methyltransferases between tumor and normal, we used a TCGA dataset with provisional version $(n=1,222$ in tumor and $n=113$ in normal samples). To assess the association between BRC patient clusters and molecular/histological subtypes, another version of TCGA dataset $(n=817$, TCGA cohort) providing clinicopatholical data was used (17). We also obtained gene expression datasets of patients with BRC from the University of North Carolina Lineberger Comprehensive Cancer Center ( $n=500$, UNC500 cohort) and the Koo Foundation SYS Cancer Center (GSE20685, n=327, KFSYSCC cohort), which are freely available from the NCBI GEO database. Among the cohorts, gene expression data from the UNC500 cohort were generated by combining 4 subpatient cohorts, including GSE18229, GSE20624, GSE2741 and GSE6128. All gene expression data used in this study contained information on patient survival and follow-up times, which was used to estimate the prognostic relevance of a gene expression signature. Recurrence-free survival was defined as the time from surgery to the first confirmed relapse. Metastasis-free survival was used as the time from surgery to the metastasis to distant organ. The baseline characteristics of the $3 \mathrm{BRC}$ patient cohorts for verifying the prognostic relevance of EHMT2 are presented in Table I.
Immunohistochemical staining. The EnVision+ kit/HRP kit (Dako, Carpinteria, CA, USA) was used for immunohistochemical staining. Paraffin-embedded sections of breast tumor specimens were processed in a microwave $\left(90^{\circ} \mathrm{C}\right)$ with an antigen-retrieval solution ( $\mathrm{pH} 9, \mathrm{~S} 2367$; Dako) and treated with a peroxidase-blocking reagent followed by a protein-blocking reagent (K130, X0909; Dako). Tissue sections were incubated $\left(4^{\circ} \mathrm{C}, 12 \mathrm{~h}\right)$ with a rabbit anti-EHMT2 antibody $(1 / 500$ dilution) (CSB-PA007497GA01HU; Cusabio, Houston, TX, USA), followed by incubation (room temperature, $1 \mathrm{~h}$ ) with an HRP-conjugated secondary antibody (K4002; Dako). Immunoreactivity was visualized with a chromogenic substrate (Liquid DAB Chromogen; Dako). Finally, tissue specimens were stained (room temperature) with Mayer's hematoxylin solution (Hematoxylin QS; Vector Laboratories) for $20 \mathrm{sec}$ to discriminate the nucleus from the cytoplasm. Human BRC tissues were purchased from Biochain Institute Inc. (T8235731-2; Newark, CA, USA)

Immunofluorescence. Immunofluorescence was performed according to previously described methods $(18,19)$. Cells grown on a 4-well chamber slide (Nalge Nunc, Rochester, NY, USA) were washed 2 times with PBS, fixed with $100 \%$ methanol for 5 min at $-20^{\circ} \mathrm{C}$. The cells were covered with PBS containing $5 \%$ bovine serum albumin for $1 \mathrm{~h}$ at room temperature to block non-specific hybridization and then incubated with rabbit anti-HSPD1 antibody (\#12165S; Cell Signaling Technology) at a 1:1,000 dilution ratio. After being washed with PBS, the cells were stained with an Alexa Fluor 488-conjugated anti-rabbit secondary antibody (1:5000 dilution ration) (A11008; Thermo Fisher Scientific, Waltham, MA, USA). Nuclei were counterstained with 4',6'-diamidino-2-phenylindole dihydrochloride (DAPI) (H-1200; Vector Laboratories, Burlingame, CA, USA).

Semi-quantitative RT-PCR. Total RNA was isolated from the indicated cell lines using a Qiagen RNeasy Mini kit according to previously described methods $(20,21)$. RNA aliquots of $1 \mu \mathrm{g}$ were then reverse transcribed using the iScript ${ }^{\mathrm{TM}} \mathrm{cDNA}$ synthesis kit (Bio-Rad Laboratories, Inc., Hercules, CA, USA) according to standard protocols. For semi-quantitative RT-PCR, cDNA was used as the template for PCR, performed using AccuPower ${ }^{\circledR}$ HotStart PCR PreMix (Bioneer, Daejeon, South Korea). For semi-quantitative RT-PCR, PCR reactions were performed using the SimpliAmp Thermal Cycler [annealing temperature $58^{\circ} \mathrm{C}, 35$ cycles (EHMT2), 30 cycles (ACTB, HSP60)] (Applied Biosystems, Foster City, CA, USA) following the manufacturer's instructions. The PCR primers used were as follows: EHMT2 (forward, 5'-GAGAACATCTGC CTGCACTG-3' and reverse, 5'-GTTGACAGCATGGAGG TCAC-3'), HSPD1 (forward, 5'-GTCTTCAGGTTGTGGCAG TC-3' and reverse, 5'-GGCATCGTCTTTGGTCACAA-3') and ACTB (forward, 5'-ACTCTTCCAGCCTTCCTTCC-3' and reverse, 5'-CAATGCCAGGGTACATGGTG-3'). Gel condition (1\% agarose gel) and Safe DNA stain for visualization method (Safe-01-01; Bioland, Scientific LLC, Paramount, CA USA) were used, and then the ATTO E-graph system (AE-9000N; ATTO, Tokyo, Japan) was used for observation.

$3 D$ culture. To perform the spheroid culturing of the MCF7 cells, ultra-low attachment microplates were used (cat. no. 4515; 
Table I. Baseline characteristics of the breast cancer patient cohorts.

\begin{tabular}{|c|c|c|c|}
\hline Variables & $\begin{array}{l}\text { UNC500 } \\
\text { cohort }\end{array}$ & $\begin{array}{c}\text { KFSYSCC } \\
\text { cohort }\end{array}$ & $\begin{array}{l}\text { TCGA } \\
\text { cohort }\end{array}$ \\
\hline Patients (n) & 500 & 327 & 817 \\
\hline \multicolumn{4}{|l|}{ Age (years) } \\
\hline Median & 55 & 46 & 59 \\
\hline Range & $24-93$ & $24-84$ & $26-90$ \\
\hline \multicolumn{4}{|l|}{ Histology $(\%)$} \\
\hline $\begin{array}{l}\text { Infiltrating ductal } \\
\text { Carcinoma }\end{array}$ & & & $599(73.3)$ \\
\hline $\begin{array}{l}\text { Infiltrating lobular } \\
\text { Carcinoma }\end{array}$ & & & $143(17.5)$ \\
\hline Mucinous carcinoma & & & $14(1.7)$ \\
\hline Medullary carcinoma & & & $5(0.6)$ \\
\hline Metaplastic carcinoma & & & $3(0.4)$ \\
\hline Others & & & $54(6.6)$ \\
\hline NA & $500(100)$ & $327(100)$ & \\
\hline \multicolumn{4}{|l|}{$\mathrm{T}$ classification } \\
\hline $\mathrm{T} 1$ & & $101(30.9)$ & $219(26.8)$ \\
\hline $\mathrm{T} 2$ & & $188(57.5)$ & $459(56.2)$ \\
\hline $\mathrm{T} 3$ & & $26(8)$ & 105 (12.9) \\
\hline $\mathrm{T} 4$ & & $12(3.7)$ & $34(4.2)$ \\
\hline NA & $500(100)$ & & \\
\hline \multicolumn{4}{|l|}{$\mathrm{N}$ classification } \\
\hline N0 & $182(36.4)$ & 137 (41.9) & $382(46.8)$ \\
\hline N1 & $189(37.8)$ & 87 (26.6) & $278(34)$ \\
\hline $\mathrm{N} 2$ & $26(5.2)$ & $63(19.3)$ & $85(10.4)$ \\
\hline N3 & $0(0)$ & $40(12.2)$ & $58(7.1)$ \\
\hline NA & 103 (20.6) & & $14(1.7)$ \\
\hline \multicolumn{4}{|l|}{ AJCC stage } \\
\hline I & & & $140(17.1)$ \\
\hline II & & & $461(56.4)$ \\
\hline III & & & $184(22.5)$ \\
\hline IV & & & 13 (1.6) \\
\hline NA & $500(100)$ & $327(100)$ & $19(2.3)$ \\
\hline \multicolumn{4}{|l|}{ ER } \\
\hline Positive & 243 (48.6) & $206(63)$ & $593(72.6)$ \\
\hline Negative & 243 (48.6) & $121(37)$ & $174(21.3)$ \\
\hline NA & $14(2.8)$ & & $50(6.1)$ \\
\hline \multicolumn{4}{|l|}{ PR } \\
\hline Positive & & 258 (78.9) & $522(63.9)$ \\
\hline Negative & & $69(21.1)$ & $251(30.7)$ \\
\hline NA & $500(100)$ & & $44(5.4)$ \\
\hline \multicolumn{4}{|l|}{ HER2 } \\
\hline Positive & 78 (15.6) & 78 (23.9) & $121(14.8)$ \\
\hline Negative & $369(73.8)$ & $249(76.1)$ & 417 (51) \\
\hline NA & $53(10.6)$ & & $279(34.1)$ \\
\hline \multicolumn{4}{|l|}{$\begin{array}{l}\text { Adjuvant } \\
\text { chemotherapy }\end{array}$} \\
\hline Yes & & $268(82)$ & 40 (4.9) \\
\hline No & & $54(16.5)$ & $12(1.5)$ \\
\hline NA & $500(100)$ & $5(1.5)$ & 765 (93.6) \\
\hline
\end{tabular}

Table I. Continued.

\begin{tabular}{|c|c|c|c|}
\hline Variables & $\begin{array}{l}\text { UNC500 } \\
\text { cohort }\end{array}$ & $\begin{array}{c}\text { KFSYSCC } \\
\text { cohort }\end{array}$ & $\begin{array}{l}\text { TCGA } \\
\text { cohort }\end{array}$ \\
\hline Death, $\mathrm{n}$ & 91 & 83 & 120 \\
\hline $\begin{array}{l}\text { Median follow-up } \\
\text { (month) }\end{array}$ & 30.5 & 97.2 & 28.9 \\
\hline
\end{tabular}

NA, not available; ER, estrogen receptor; PR, progesterone receptor; human epidermal growth factor receptor 2 .

Corning Inc., Corning, NY, UYSA). Following EHMT2 knockdown, $1 \times 10^{4}$ cells were loaded onto a spheroid culture plate, grown for 3 days, and then observed under a microscope(Ti-S; Nikon, Tokyo, Japan), as previously described (22).

siRNA transfection. siRNA duplexes against EHMT2 (siEHMT2; 5'-GCAAAUAUUUCACCUGCCATT-3' and 5'-UGGCAGGUGAAAUAUUUGCTT-3') were purchased from ST Pharm Co. Ltd. (Seoul, Korea), and siRNA duplexes against HSPD1 (siHSPD1; 5'-GUGUUGAAGGAUCUU UGAUTT-3' and 5'-AUCAAAGAUCCUUCAACACTT-3') were purchased from Bioneer (Daejeon, Korea). Negative control siRNA (siCont; 5'-AUGAACGUGAAUUGCUCA ATT-3' and 5'-UUGAGCAAUUCACGUUCACTT-3') was used for the control treatments. The siRNAs (100 nM) were transfected into the cancer cell lines using RNAiMax (Thermo Fisher Scientific, Inc.) for $72 \mathrm{~h}$.

Western blot analysis. The cells were washed once with PBS and then lysed in cold lysis buffer $(50 \mathrm{mM}$ Tris-HCl, $\mathrm{pH} 7.4$, $150 \mathrm{mM} \mathrm{NaCl}, 1 \%$ Triton $\mathrm{X}-100,0.1 \%$ SDS, 1 mM EDTA, $1 \mathrm{mM} \mathrm{Na} 3 \mathrm{VO} 4,1 \mathrm{mM} \mathrm{NaF}$ and $1 \mathrm{X}$ protease inhibitor cocktail). Cell lysates were centrifuged at $14,000 \times \mathrm{g}$ for $15 \mathrm{~min}$ at $4^{\circ} \mathrm{C}$ and then boiled in $5 \mathrm{X}$ sample buffer following protein determination (BSA, \#23208; Thermo Fisher Scientific, Inc.). The protein samples were subjected to western blot analysis. For western blot analysis, nitrocellulose membranes (\#1620145; Bio-Rad Laboratories, Inc.) and blocking reagent (5\% skim milk, $1 \mathrm{~h}$, room temperature), precasting gel (\#456-1094; Bio-Rad Laboratories, Inc.) were used with the indicated antibodies at a 1:1,000 or 1:500 dilution ratio. The samples were stained with anti-HSPD1 (\#12165S), poly(ADP-ribose) polymerase (PARP; \#9542S) and caspase-3 (\#9662S) antibodies from Cell Signaling Technology, Inc. (Danvers, MA, USA), EHMT2 (CSB-PA007497GA01HU) antibodies from Cusabio and ACTB (SC-47778) antibodies from Santa Cruz Biotechnology, Inc. (Santa Cruz, CA, USA), at $4^{\circ} \mathrm{C}$ (overnight). Secondary antibodies (rabbit; SC-2357, mouse; SC-2031, Santa Cruz Biotechnology, Inc.) were incubated at room temperature, $1 \mathrm{~h}$, and ECL solution (\#170-5060; Bio-Rad Laboratories, Inc.) was used for visualization.

Cell growth assay. Following treatment, the cells were washed twice with PBS and fixed with cold $100 \%$ methanol for $5 \mathrm{~min}$ at $-20^{\circ} \mathrm{C}$. After being washed twice with PBS, the cells were stained with $0.1 \%$ crystal violet solution $(\mathrm{C} 0775$; 
Sigma-Aldrich; Merck KGaA, Darmstadt, Germany) for 5 min at room temperature. The cells were then washed 5 times with distilled water and observed under a microscope (Ti-S; Nikon). The results were analyzed using ImageJ software (version 1.8.0).

Statistical analysis. To divide the BRC patients into 2 groups based on EHMT2 gene expression, we applied the median gene expression value in each cohort as the cut-off. The Kaplan-Meier method was used to calculate the time to death, metastasis, or recurrence and differences between the times were assessed using log-rank statistics. A hierarchical clustering algorithm was applied using the centered correlation coefficient as the measure of similarity and complete linkage clustering. To estimate the independent utility of the signature, we performed multivariate Cox regression analysis combined with known clinicopathological risk factors. A backward-forward step procedure (function step, $\mathrm{R}$ package stats) was carried out to optimize the multivariate model with the most informative variables. For comparing the ratio of molecular or histological factors between the subpatient groups, we applied Fisher's exact ( 2 categories) or $\chi^{2}$ tests ( 3 or more categories). To assess the associations between mRNA expression and the prognostic subgroups divided by the signature, we performed point-biserial correlation tests (function cor.test, $\mathrm{R}$ package stats)on the expression data and BRC patient subgroups. To estimate the significance of gene expression differences between the patient subgroups, we performed a two-sample t-test for each gene. All statistical tests were carried out using $\mathrm{R}$ language environment (ver. 3.5.1).

Gene set enrichment and upstream regulator analyses were performed using Ingenuity Pathway Analysis (IPA, Ingenuity Systems, www.ingenuity.com). Gene set enrichment analysis was carried out to identify the most significant gene sets associated with the disease process, molecular and cellular functions, and physiological and developmental conditions. The significances of over-represented gene sets were estimated by Fisher's exact test. To explore the associations between genes in the gene set associated with EHMT2, we performed upstream regulator analysis searching for known targets of each regulator in the dataset and compared their direction of change to the expected change based on previously published literature provided by IPA knowledgebase.

\section{Results}

Overexpression of EHMT2 in BRC. To identify histone methyltransferase overexpression, we gathered RNA-seq data on normal (113 samples) and BRC (1,222 samples) tissues from the TCGA portal and analyzed the expression levels of 61 histone methyltransferase and demethylase proteins, revealing an upregulated EHMT2 expression in BRC tissues compared with that in normal tissues (Fig. 1A and B). Moreover, immunohistochemical analysis with the BRC tissue microarray revealed a high EHMT2 expression in BRC tissues compared with that in normal breast tissue (Fig. 1C). Taken together, these results indicated that EHMT2 was overexpressed in BRC.

To identify the connection between EHMT2 and cell growth, we performed a cell growth assay following transfection with siEHMT2; siCont served as the negative control. EHMT2 expression was significantly decreased by transfection with siEHMT2 (Fig. 1D, left panel), and in the growth assay, the number of cells was suppressed by EHMT2 knockdown (Fig. 1D, right panel). In addition, we detected cleaved caspase-3 and PARP expression following transfection with siEHMT2 in BRC cell lines by western blot analysis. Our results suggested that the overexpression of EHMT2 may promote cell proliferation via inhibiting cell apoptosis, as the knockdown of EHMT2 increased the levels of cleave PARP and caspase-3 (Fig. 1E). Subsequently, to assess whether EHMT2 affects cell aggregation, we performed 3D culturing, a biomimetic system for cancer cell growth, and observed inhibited cell aggregation in the EHMT2 knockdown group compared with that in MCF7 cells transfected with siCont (Fig. 1F). Thus, EHMT2 plays important roles in the survival and proliferation of BRC cell lines.

Prognostic value of EHMT2 in patients with BRC. To determine whether EHMT2 has prognostic value in BRC, we analyzed gene expression data obtained from independent BRC patient cohorts. When dividing patients in the UNC500 cohort into 2 groups based on the median threshold of EHMT2 expression, the overall survival rate was significantly lower in the high-EHMT2 subgroup than in the low-EHMT2 subgroup (log-rank test, $\mathrm{P}<0.001$; Fig. 2A and B). When estimating recurrence-free survival with the same cut-off value, the recurrence rate in the high-EHMT2 subgroup was also significantly higher than that in the low-EHMT2 subgroup (log-rank test, $\mathrm{P}=0.002$; Fig. 2C). By applying the same procedure to the KFSYSCC cohort, a consistent statistical significance in the prediction of overall survival was obtained (log-rank test, $\mathrm{P}=0.039$; Fig. 2D). Since metastasis-free survival data were available for the KFSYSCC cohort, we also applied the same cut-off value to those data and assessed its prognostic value. When estimating metastasis-free survival using EHMT2 expression in the KFSYSCC cohort, however, we did not found a significant difference between the 2 groups, but rather a tendency towards correctly classifying high-risk BRC patients by EHMT2 expression (Fig. 2E), indicating a limitation of using a single gene as a diagnostic tool. Although we also performed a survival analysis to estimate the prognostic value of EHMT2 in patients with BRC from the TCGA cohort, no significant differences in survival were obtained (data not shown). This result may have been due to a limitation of the TCGA cohort, in which the sample composition was inadequate for a prognostic prognosis (23), although many samples were included in the cohort.

EHMT2-related genes involved in the cell apoptotic process. We then performed RNA-seq analysis following the knockdown of EHMT2 to investigate EHMT2-related cellular pathways, identifying 1,765 differentially expressed genes (DEGs; 911 downregulated and 854 upregulated). Functional enrichment analysis using IPA software revealed that the cell cycle, cell death and survival, cellular growth and proliferation pathways were significantly enriched (Fig. 3A). Moreover, a network-based analysis of GO terms (biological processes) revealed that the mitotic cell cycle process $(44 \%)$, cellular 

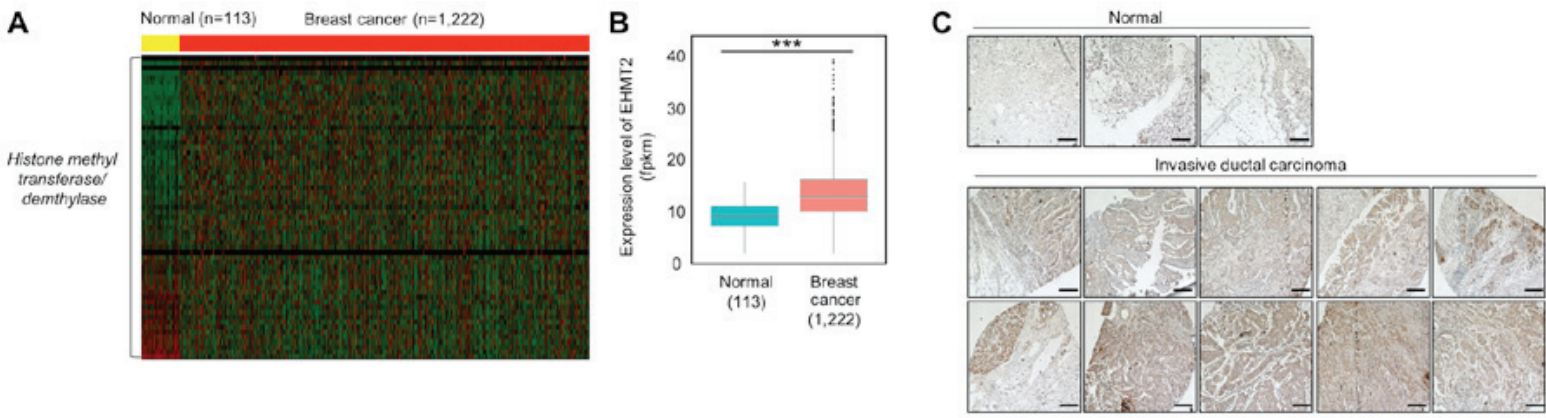

D

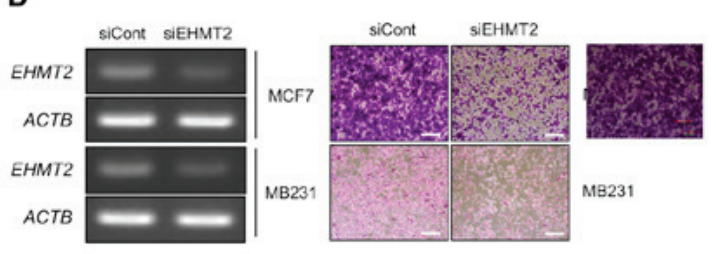

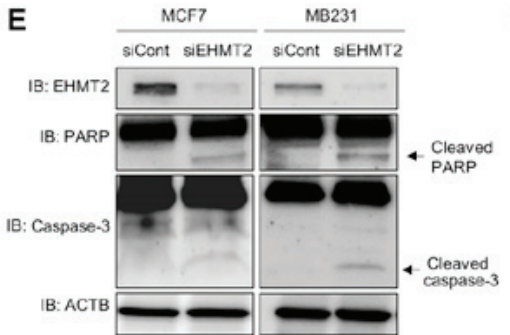

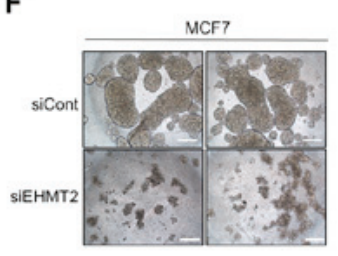

Figure 1. EHMT2 is overexpressed in breast cancer (BRC). (A) Heatmap of gene expression related to histone methylation/demethylation in the in silico histone methyltransferase/demethylase panel, sorted by fold change in the BRC/normal FPKM value. In the heatmap, yellow indicates normal liver samples, while red indicates BRC samples in TCGA. The threshold was set to a 2.0-fold-change. (B) Expression levels of EHMT2 in BRC using normal and BRC samples. P-values were calculated using Wilcoxon's tests $\left.{ }^{(* * *} \mathrm{P}<0.001\right)$. (C) Immunohistochemical analysis of EHMT2 in a BRC tissue microarray. Breast cancer tissues were purchased from BioChain. Scale bar, $200 \mu \mathrm{m}$. (D) Cell growth assay. After knocking down EHMT2 for $72 \mathrm{~h}$, RT-qPCR analysis of EHMT2 was performed. Actin (ACTB) was used as the internal control (left panel). Cell fixation with $100 \%$ methanol and cell staining with crystal violet was performed. Scale bar, $100 \mu \mathrm{m}$ (right panel); siRNA duplexes against EHMT2 (siEHMT2; 5'-GCAAAUAUUUCACCUGCCATT-3', 5'-UGGCAGGUGAAAUAUUUGCTT-3') were purchased from ST Pharm. Negative control siRNA was also used (siCont; 5'-AUGAACGUGAAUUGCUCAATT-3', 5'-UUGAGCAAUUCACGUUCACTT-3'). (E) Western blot analysis of EHMT2, PARP, caspase-3 and $\beta$-catenin. ACTB was used as the internal control. (F) Spheroid formation. MCF7 cells treated with siEHMT2 and siCont were loaded onto a spheroid plate and incubated for $96 \mathrm{~h}$. The cells were imaged under a microscope. Scale bar, $500 \mu \mathrm{m}$.
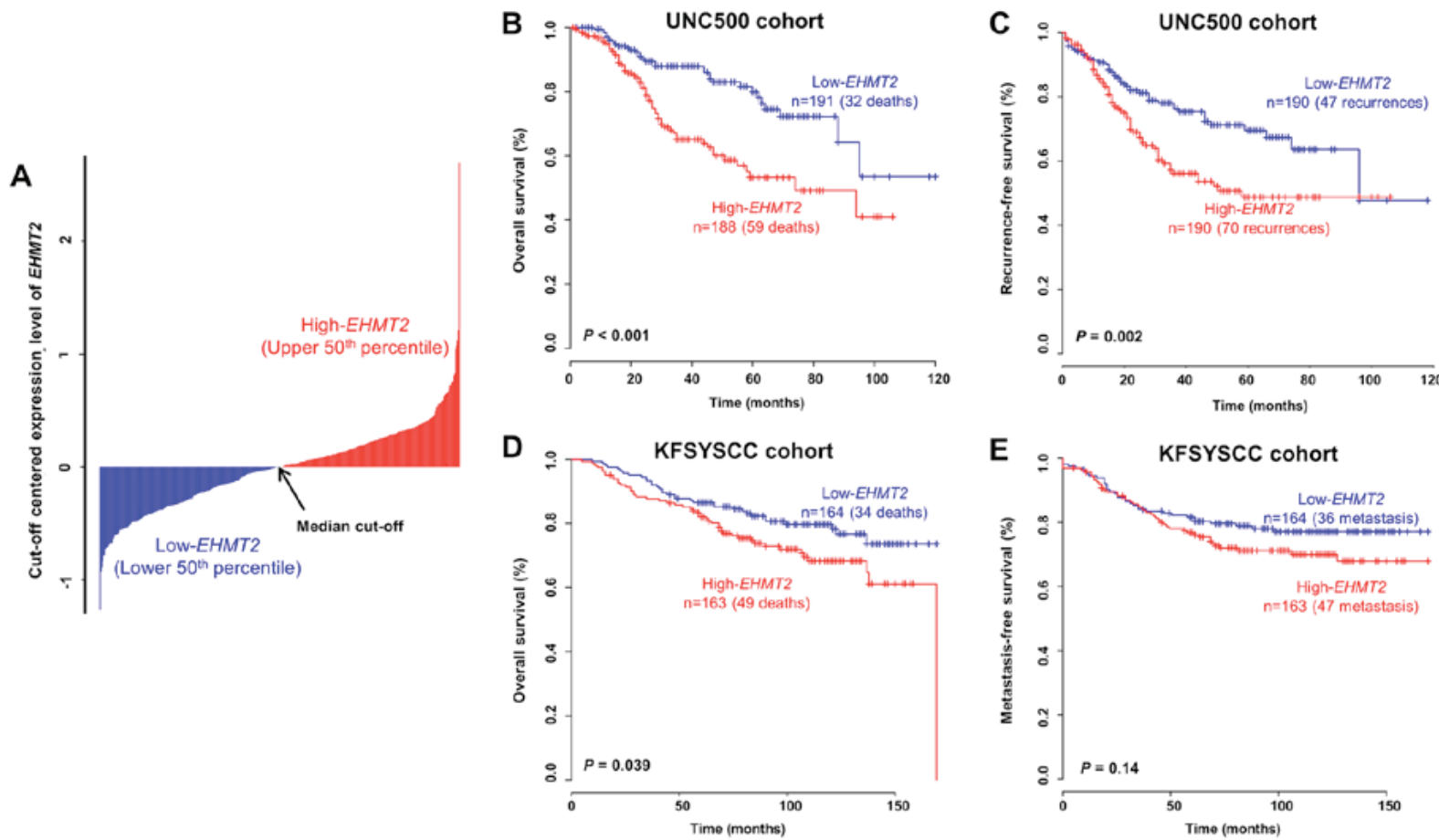

Figure 2. EHMT2 expression and breast cancer (BRC) prognosis. (A) Expression of EHMT2 in patients with BRC from the UNC500 cohort. The median value of EHMT2 expression was considered the cut-off in the patient cohort. (B and C) Kaplan-Meier curves of (B) overall survival and (C) recurrence-free survival in the UNC500 cohort. (D and E) Kaplan-Meier curves of (D) overall survival and (E) metastasis-free survival in the KFSYSCC cohort.

response to stress process $(12 \%)$ and apoptotic process $(10 \%)$ were represented using ClueGO (plugged into Cytoscape) (Fig. 3B). Thus, as shown in Fig. 3, genes regulated by EHMT2 are involved in cell proliferation processes, such as cell apoptosis, the cell cycle and cellular response to stress, implying that EHMT2 can be a molecular target for BRC therapy. 
A

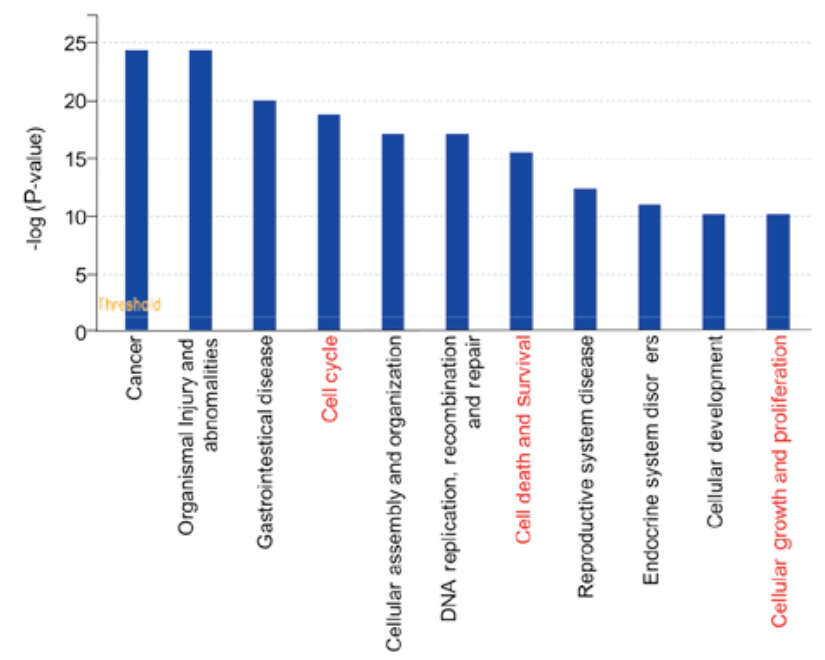

B
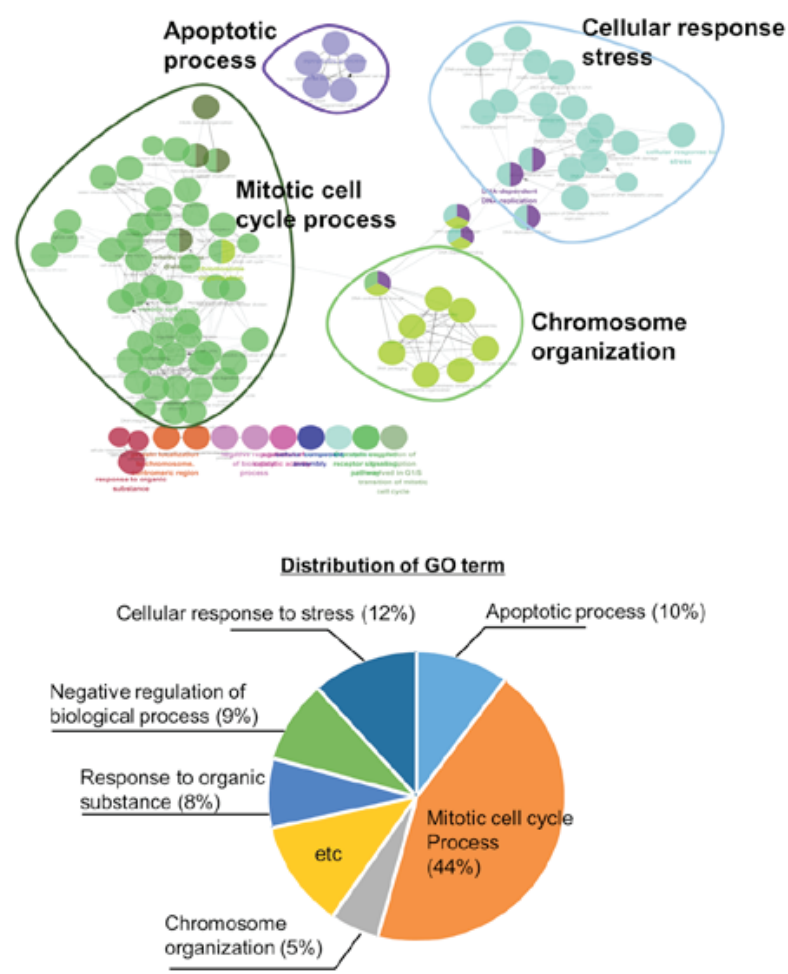

Figure 3. RNA-seq analysis after siEHMT2 treatment. (A) Functional enrichment analysis of RNA-seq EHMT2 knockdown results obtained from the Ingenuity Pathway Knowledge Base. (B) Gene Ontology (GO) pathway term enrichment networks. GO pathway term networks in the EHMT2 knockdown and control groups were functionally grouped by ClueGO (top panel). The Venn diagram shows the distribution of the functionally grouped GO terms (bottom panel). Terms in the functionally grouped networks were cut-off at $\mathrm{P}<0.05$.

HSPD1 expression is regulated by EHMT2. To assess the signaling pathways involved in EHMT2-related cancer progression, we performed western blot analysis using a human phospho-kinase array kit (ARY003B) purchased from R\&D Systems Inc. (Minneapolis, MN, USA). We observed the same control spot intensities between the siCont and siEHMT2 groups; however, the intensity of HSPD1 expression was significantly decreased in the EHMT2 knockdown group compared to that in the siCont group (Fig. 4A). In addition, the expression levels of HSPD1 were increased in the BRC samples, as similar to EHMT2 expression (Fig. 4B). Furthermore, RNA-seq and RT-PCR analyses revealed that HSPD1 expression was decreased following transfection with siEHMT2 at the transcriptional level (Fig. 4C and D, upper panel). We also confirmed the downregulation of HSPD1 expression by EHMT2 knockdown using western blot analysis (Fig. 4D, lower panel).

HSPD1 is a molecular chaperone that assists with protein folding, tracking and degradation and is associated with the enhancement of tumor cell survival via the intrinsic apoptotic pathway (13). Thus, to assess whether HSPD1 knockdown induces the apoptosis of BRC cells, we performed cell growth analysis following transfection with siHSPD1. The cell numbers in the siHSPD1-transfected group were significantly decreased compared with those in the siCont group (Fig. 4E). Moreover, the levels of cleaved PARP and caspase- 3 were clearly detected in the siHSPD1 group (Fig. 4F). Thus, the downregulation of HSPD1 expression by EHMT2 knockdown activated the cell apoptotic pathway.
The subcellular localization of HSPD1 is associated with protein functions, such as pro-apoptosis and anti-apoptosis, in cancer (24). As is clearly shown in Fig. 4G, HSPD1 was localized to a cytoplasmic area in the siCont group, while this cytoplasmic HSPD1 localization was markdly absent in the siEHMT2 group. Taken together, these results suggest that HSPD1 re-localization and HSPD1 expression downregulated by EHMT2 knockdown induces cell apoptosis via an HSPD1-related anti-apoptosis pathway. In other words, EHMT2 overexpression promotes BRC cell proliferation, as reflected by the elevated expression of HSPD1.

BIX01294 suppresses HSPD1 expression and the induction of cell apoptosis. BIX01294 is a specific inhibitor that downregulates EHMT2 activity $(25,26)$. To confirm whether BIX01294 treatment affects HSPD1 expression and cell survival in BRC cell lines, we performed cell growth and biochemical analyses following BIX01294 treatment. The cell numbers in the BIX01294-treated group were significantly decreased compared to those in the siCont group, and HSPD1 expression was also downregulated by BIX01294 treatment similar to the siEHMT2 treatment group (Fig. $4 \mathrm{H}$ and I). In addition, the levels of cleaved PARP and caspase-3 were clearly detected in the EHMT2 knockdown group (Fig. 4J). Taken together, these results suggest that the development of EHMT2-specific inhibitors is crucial for BRC therapy.

Prognostic relevance of EHMT2 regulatory genes. To explore genes regulated by EHMT2 activity, we selected genes 
A

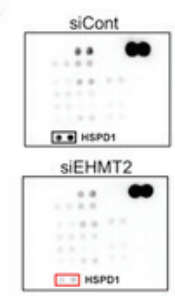

B

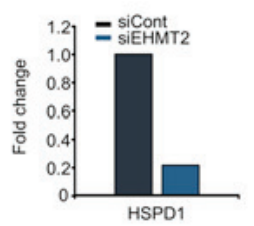

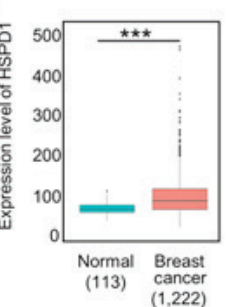

C

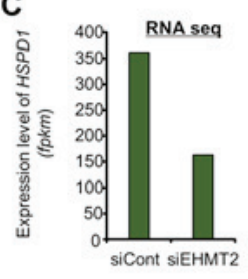

D

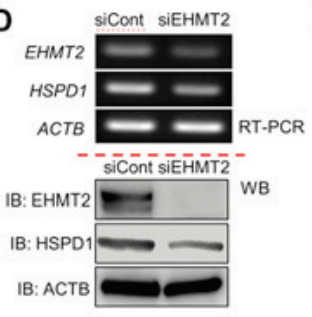

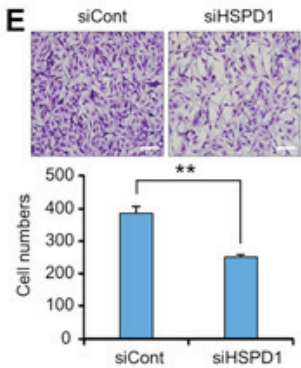

$\mathbf{F}$

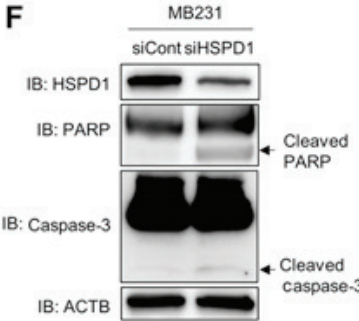

G

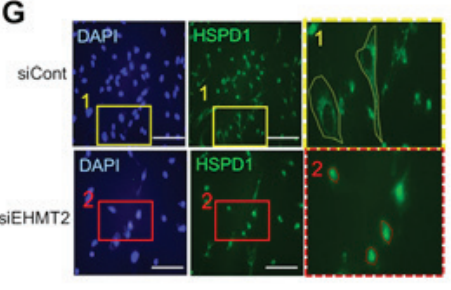

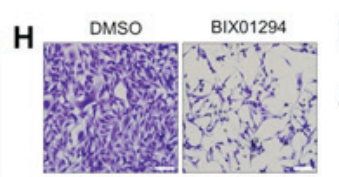

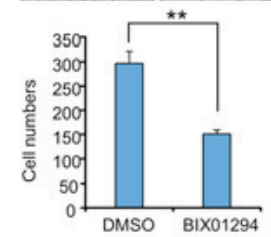

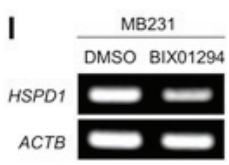

ACTB

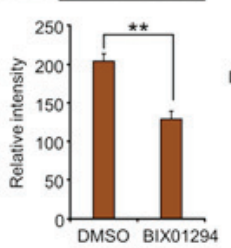

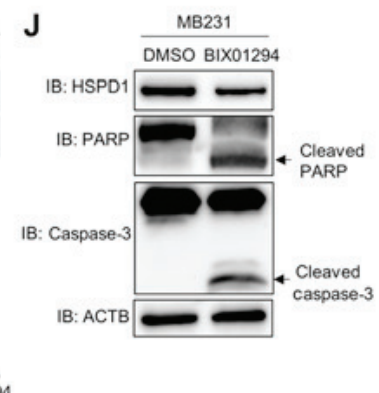

Figure 4. EHMT2 controls the expression and subcellular localization of HSPD1. (A) Phosphor array with the lysates of cells in which EHMT2 was knocked down. The phosphor array (ARY003B) was purchased from R\&D Biosystems. Approximately $200 \mu \mathrm{g}$ of cell lysate was used. (B and C) Expression levels of HSPD1 in breast cancer samples (TCGA); P-values were calculated using Wilcoxon's tests $\left({ }^{* * *} \mathrm{P}<0.001\right)(\mathrm{B})$ and RNA-seq results (C). (D) RT-qPCR analysis of HSPD1 after EHMT2 knockdown in MDA-MB-231 cell lines. ACTB was used as the internal control (top panel). Western blot analysis was performed using the indicated antibodies (bottom panel). (E) Cell growth assay. After knocking down HSPD1 for $72 \mathrm{~h}$, the cells were fixed with $100 \%$ methanol and stained with crystal violet. Scale bar, $200 \mu \mathrm{m}$ (top panel); P-values were calculated using Student's t-tests $\left({ }^{* *} \mathrm{P}<0.01\right)($ bottom panel). (F) Western blot analysis after HSPD1 knockdown using anti-HSPD1, anti-PARP, anti-caspase3 and anti- $\beta$-catenin antibodies. ACTB was used as the internal control in MB231 cells. (G) Immunocytochemical analysis of HSPD1. MB231 cells treated with siCont or siEHMT2 were fixed with $100 \%$ methanol and stained with anti-HSPD1 (Alexa Fluor 488, green) and DAPI (blue). Scale bar, $200 \mu \mathrm{m}$. (H) Cell growth assay. After MB231 cells were treated with BIX01294 for 24 h, the cells were fixed with $100 \%$ methanol and stained with crystal violet. Scale bar, $200 \mu \mathrm{m}$ (top panel); P-values were calculated using Student's t-tests $\left({ }^{* *} \mathrm{P}<0.01\right)$. (I) RT-qPCR analysis of HSPD1 after treatment with BIX01294. ACTB was used as the internal control (top panel), and the signal intensities corresponding to HSPD1 were quantified using ImageJ software (bottom panel). The P-values were calculated using Student's t-test ( $\left.{ }^{* *} \mathrm{P}<0.01\right)$. (J) Western blot analysis after treatment with BIX01294 using anti-HSPD1, anti-PARP, anti-caspase 3 and anti- $\beta$-catenin antibodies. ACTB was used as the internal control in MB231 cells.

A

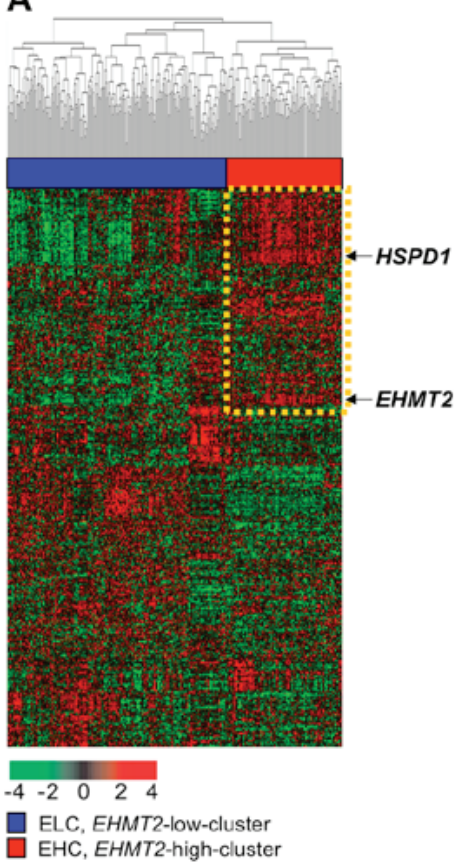

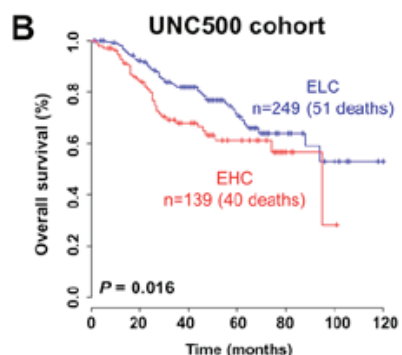
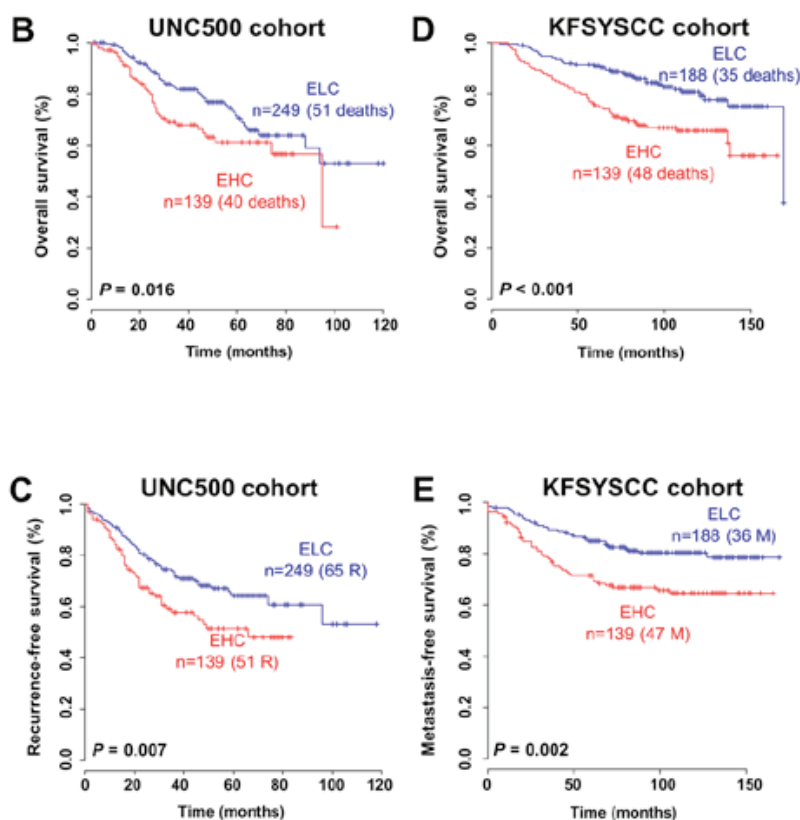
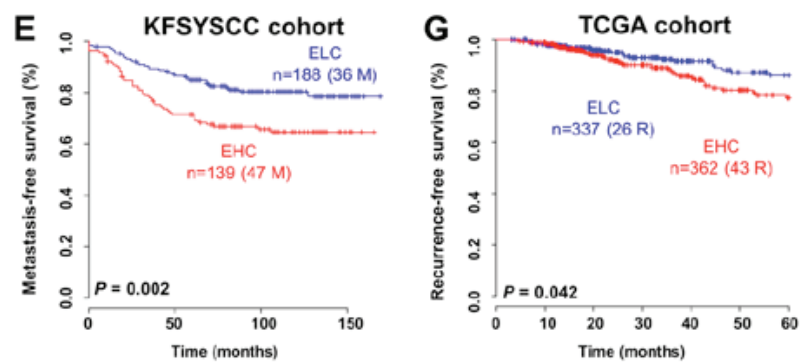

Figure 5. Gene expression pattern of the EHMT2 signature and patient survival of two clusters from the UNC500, KFSYSCC and TCGA cohorts ( $\mathrm{n}=388$, 327 and 775, respectively). Follow-up time and survival data were used in this analysis. (A) Gene expression patterns of EHMT2 and its associated genes in the UNC500 cohort. A total of 1,765 genes having at least a 2-fold difference between siEHMT2 and siCont cells were involved in the EHMT2 signature. Among these genes, 1,410 were used in the hierarchical clustering analysis of the UNI500 cohort. The patients were divided into 2 groups: an EHMT2-low cluster group (ELC) and an EHMT2-high cluster group (EHC). (B and C) Kaplan-Meier plot depicting (B) overall and (C) recurrence-free survival rates. The survival rates of the patients in the EHC group were significantly increased compared with those of the patients in the ELC group (each $\mathrm{P}<0.05$ as determined by log-rank test). (D-G) Several survival rates of the two patient subgroups in the (D and E) KFSYSCC and (F and G) TCGA cohorts. 


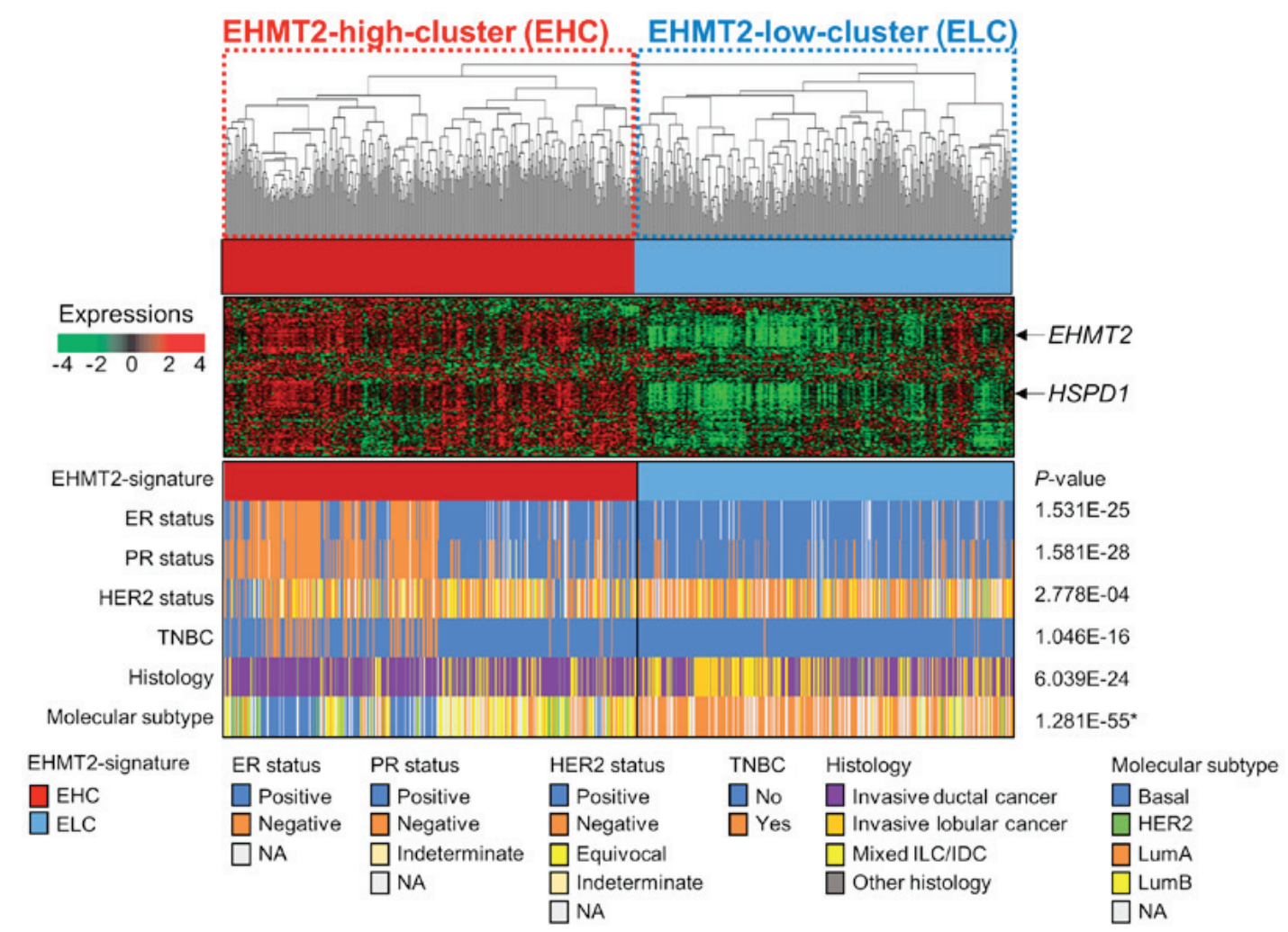

Figure 6. Gene expression pattern of the EHMT2 signature and its association with molecular features in the TCGA cohort (n=817). Histological and molecular subtypes of breast cancer displayed according to the patient clusters divided by EHMT2 signature. ${ }^{*}$ P-value was obtained by an $\chi^{2}$ test and the remaining P-values were obtained by Fisher's exact tests.

that were differentially expressed between the siCont- and siEHMT2-treated MCF7 cell lines. A total of 1,765 genes exhibiting at least a 2 -fold difference in expression were observed. We defined a signature consisting of these genes that interacts with EHMT2, named the EHMT2 signature, and sought to verify its prognostic relevance in BRC. When applying the EHMT2 signature to gene expression data from the UNIC500 cohort, 1,410 common gene features were observed. Based on hierarchical clustering analysis of the expression patterns of the signature in the UNC500 cohort, we divided the BRC samples into 2 subgroups as follows: an EHMT2-high cluster (EHC) subgroup and an EHMT2-low cluster (ELC) subgroup (Fig. 5A). The overall survival rate of the patients in the EHC group was significantly lower than that of the patients in the ELC group (log-rank test, $\mathrm{P}=0.016$; Fig. 5B), and the recurrence rate in the EHC subgroup was significantly higher than that in the ELC subgroup (log-rank test, $\mathrm{P}=0.007$; Fig. 5C). To validate the observations in the UNC500 cohort, we analyzed gene expression data from the KFSYSCC cohort. Using the same procedure employed with the UNC500 cohort, the patients in the KFSYSCC cohort were divided into 2 subgroups (EHC and ELC) by hierarchical clustering analysis using the 1,604 genes that overlapped the EHMT2 signature (data not shown). The overall and metastasis-free survival rates of each group were estimated. Kaplan-Meier analysis revealed that the EHMT2 signature was a significant predictor of overall and metastasis-free survival of patients with BRC in the KFSYSCC cohort (log-rank tests, $\mathrm{P}<0.001$ and $\mathrm{P}=0.002$ for overall and metastasis-free survival, respectively; Fig. 5D and E). When applying the same clustering algorithms and Kaplan-Meier analysis to the TCGA cohort with the 1,644 overlapping genes, a clear tendency towards classifying high-risk patients with BRC for overall survival was observed, although statistical significance was not achieved (log-rank test, $\mathrm{P}=0.1$; Fig. 5F). However, the recurrence rate of the $\mathrm{EHC}$ subgroup classified by the EHMT2 signature was significantly higher than that of the ELC subgroup in the TCGA cohort (log-rank test, $\mathrm{P}=0.042$; Fig. 5G).

As the EHMT2 signature exhibited limited prognostic value in the TCGA cohort, we explored associations between the signature and known molecular and histological subtypes of BRC. We stratified the BRC samples from the TCGA cohort into the EHC and ELC subgroups by the EHMT2 signature and examined EHMT2 signature-associated molecular and histological subtypes (Fig. 6). When comparing the ER, PR and HER2 status between the EHC and ELC subgroups, the number of patients with BRC with a negative status of each marker was significantly higher in the EHC subgroup than in the ELC subgroup (Fisher's exact tests, both $\mathrm{P}<0.001$, Fig. 6). Regarding the patients with triple negative BRC (TNBC), their frequency in the EHC subgroup was much higher than that in the ELC subgroup (Fisher's exact test, $\mathrm{P}<0.001$, Fig. 6), consistent with the poor prognosis of patients in the EHC subgroup (Fig. 6). The exploration of histological subtypes across EHMT2 signature-based subgroups revealed that many patients with invasive lobular cancer were present in the ELC subgroup, whereas most of the patients in the EHC subgroup had invasive ductal cancer (Fisher's exact test, $\mathrm{P}<0.001$, Fig. 6). The comparisons of known molecular subtypes of BRC with the EHMT2 signature revealed that many BRC samples of the 
Table II. Univariate and multivariate Cox regression analysis of overall survival in breast cancer (combined with the UNC500 and KFSYSCC cohorts).

\begin{tabular}{|c|c|c|c|c|c|c|}
\hline \multirow[b]{2}{*}{ Variable } & \multicolumn{3}{|c|}{ Univariate } & \multicolumn{3}{|c|}{ Multivariate } \\
\hline & $\mathrm{n}$ & $\mathrm{HR}(95 \% \mathrm{CI})$ & P-value & $\mathrm{n}$ & $\mathrm{HR}(95 \% \mathrm{CI})$ & P-value \\
\hline Age $(>35$ years or not $)$ & 706 & $1.149(0.624-2.117)$ & 0.655 & 706 & & \\
\hline Node status (positive or negative) & 706 & $1.766(1.276-2.443)$ & $<0.001$ & & $1.795(1.296-2.487)$ & $<0.001$ \\
\hline ER status (positive or negative) & 706 & $0.493(0.364-0.668)$ & $<0.001$ & & $0.59(0.421-0.826)$ & 0.002 \\
\hline HER2 status (positive or negative) & 706 & $1.471(1.054-2.052)$ & 0.023 & & & \\
\hline EHMT2 signature (LEC vs. HEC ${ }^{a}$ ) & 706 & $1.726(1.281-2.326)$ & $<0.001$ & & $1.423(1.018-1.988)$ & 0.039 \\
\hline
\end{tabular}

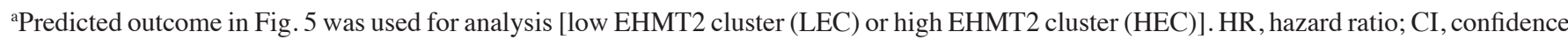
interval.

A
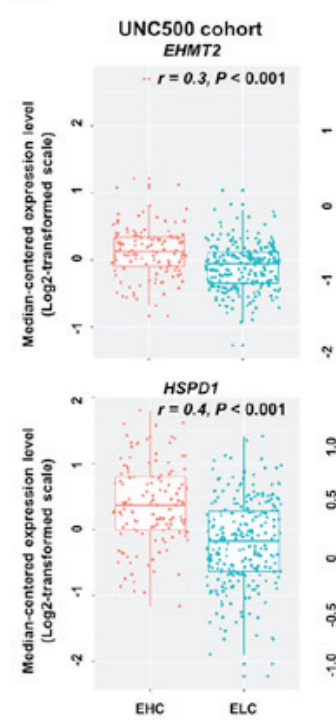
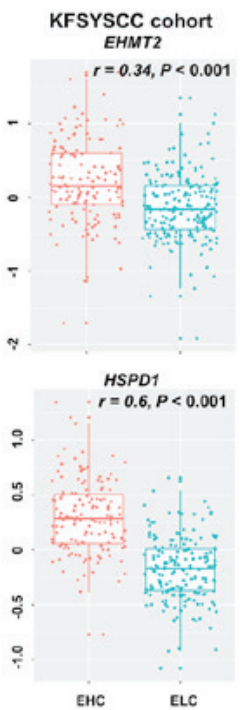

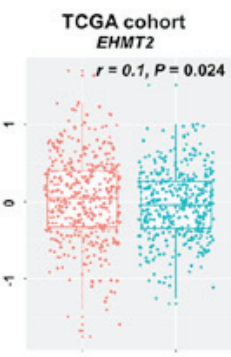

HSPD1

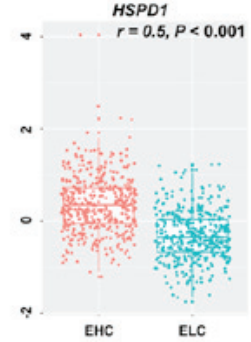

B

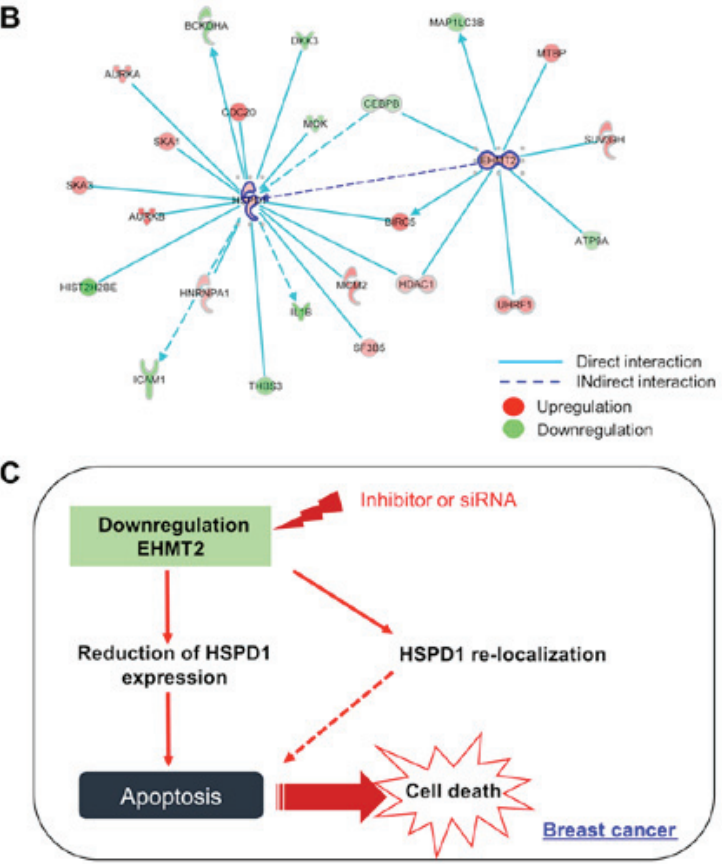

Figure 7. Association between EHMT2 and HSPD1 in the breast cancer (BRC) clinical cohorts. (A) Comparison of expression levels between the patients in the EHC and ELC groups. A two-group box plot comparing the expression levels of EHMT2 and HSPD1 in EHC and ELC patients is illustrated. P-values were obtained by two-sample t-tests between the EHC and ELC subgroups. The $r$ value indicates the correlation coefficient value of the gene compared with the EHC and ELC subgroup categories. (B) Gene-to-gene networks of EHMT2 and HSPD1 associated with BRC prognosis. Up- and down-regulated genes in the high-EHMT2 cluster (EHC) group are indicated in red and green, respectively. The intensity of the color is indicative of the degree of over- or under-expression. Each line and arrow represent functional and physical interactions between the genes and the direction of regulation reported in the literature, respectively. (C) Schematic summary of the effects of EHMT2 on breast cancer.

LumA subtype $(77.3 \%, 235$ out of 304$)$ were classified into the ELC subgroup, whereas the vast majority of BRC samples of the Basal subtype were stratified into the EHC subgroup (99\%, 106 out of 107). The difference in molecular subtypes between the EHC and ELC subgroups was statistically significant ( $\chi^{2}$ test, $\mathrm{P}<0.001$, Fig. 6 ). Taken together, these results suggest that the EHMT2 signature well reflects distinct molecular and histological subtypes of BRC and has predictive ability for classifying high-risk BRC patients, such as patients with TNBC.

To determine the prognostic independence of the newly identified EHMT2 signature, we combined clinical data from 2 cohorts (UNC500 and KFSYSCC cohorts) in which the EHMT2 signature displayed distinct predictive value regarding overall survival. We then applied Cox regression analyses to our signature and known clinicopathological risk factors. In the univariate analysis, significant prognostic indicators of BRC overall survival contained the node, ER and HER2 status along with the EHMT2 signature (Table II). When the multivariate test was performed on the combined cohort, the EHMT2 signature retained its statistical significance for overall BRC patient survival even after a variable selection procedure was applied $(\mathrm{HR}=1.423,95 \% \mathrm{CI}$ : 1.018-1.988, $\mathrm{P}=0.039$; Table II), demonstrating the prognostic 
relevance of the EHMT2 signature as an independent risk factor for BRC.

Activated regulators in the prognostic EHMT2 regulatory gene network. To confirm the alteration in HSPDI expression by EHMT2 activity, we compared their expression levels in the clinical sample subgroups (EHC and ELC). The EHMT2 and $H S P D 1$ expression levels in the EHC subgroup were significantly higher than those in the ELC subgroup of the UNC500 cohort. The expression levels of the two genes strongly correlated with the EHC and ELC subpatient groups (Fig. 7A). When comparing the expression levels of EHMT2 and HSPD1 in the KFSYSCC cohort, we also observed significant differences between the EHC and ELC subgroups and significant positive correlations with these subgroups. Finally, in the TCGA cohort, a moderate correlation was observed between EHMT2 expression and the patient subgroups divided by the EHMT2 signature $(\mathrm{r}=0.1)$. However, the correlation still retained statistical significance $(\mathrm{P}<0.001$ by polyserial correlation test), and significant differences in expression between the EHC and ELC subgroups were observed $(\mathrm{P}<0.001$ by a two-sample t-test; Fig. 7A). HSPDI expression in the EHC subgroup was significantly higher than that in the ELC subgroup, and HSPDI expression levels profoundly correlated with the EHC and ELC subpatient groups in the TCGA cohort (Fig. 7A). Finally, gene-to-gene network analysis revealed that EHMT2 formed a gene network hub in which HSPDI was indirectly regulated by EHMT2 (Fig. 7B).

\section{Discussion}

EHMT2 is a histone methyltransferase that specifically methylates histone $\mathrm{H} 3$ lysine 9 (H3K9), which is a suppressor of gene expression at the transcriptional level. Therefore, eliminating EHMT2 expression reduces the status of $\mathrm{H} 3 \mathrm{~K} 9$ methylation, and the heterochromatin structure is subsequently altered to the open chromatin formation for gene induction (6). In this study, the results of RNA-seq analysis after EHMT2 knockdown revealed that upregulated genes were implicated as being putative direct targets of EHMT2. However, we observed that EHMT2 knockdown and treatment with a specific inhibitor (BIX01294) induced the downregulation of HSPD1 in BRC cells (Fig. 4D and I). In addition, a cohort analysis revealed statistically positive correlations between EHMT2 and HSPD1 expression (Fig. 7A). Thus, we demonstrated that HSPD1 expression was indirectly regulated by EHMT2, and an unknown target regulated by EHMT2 modulates the downregulation of HSPD1. To identify HSPD1 regulation as a direct target of EHMT2, we performed gene network analysis using IPA software with RNA-seq data resulting from EHMT2 knockdown, revealing upstream regulators of HSPD1 (Fig. 7B). However, a ChIP-assay or ChIP-seq analysis is necessary to identify the direct upstream regulators of HSPD1 regulated by EHMT2.

HSPD1 expression is critically involved in several types of cancer; for example, its expression is up-regulated in colorectal, cervical, Hodgkin lymphoma, and prostate cancer (13). In addition, low HSPD1 expression has been identified as a risk factor in recurrent bladder cancer (7). Although the expression level of HSPD1 in cancer is an important factor in cell survival and apoptosis, the localization of HSPD1 between the cytoplasm and mitochondria is also associated with both pro-apoptotic and pro-survival functions in cancer $(14,24,25)$. In this stuyd, we observed the cytoplasmic localization of HSPD1 in the siCont group; however, in the siEHMT2 group, HSPD1 localization in the cytoplasmic region was clearly absent and was instead concentrated in the endoplasmic reticulum (ER) region (Fig. 4G). Arya et al demonstrated that ER-specific HSPD1 localization correlated with cell apoptosis via the reduction of XIAP expression (24). In this study, we demonstrated that EHMT2 overexpression mediated the localization of HSPD1 as well as its expression, which affected the proliferation of BRC. Regarding re-localization, Cho et al reported that methylation of HSP70 by SETD1A changes its location from the cytoplasm to the nucleus (27). Thus, it is suggested that the direct methylation of HSPD1 by EHMT2 may affect its localization. However, additional experiments need to be conducted to confirm the HSPD1 methylation by EHMT2.

Based on the expression of EHMT2, in this study, we investigated its association with BRC patient survival. When dichotomizing the patients with BRC into two risk-predictive subgroups based on EHMT2 expression, many high-risk patients were correctly classified into the high-EHMT2 subgroup (Fig. 2B-E), indicating that EHMT2 has predictive value as a prognostic marker. Although limitations of EHMT2 as a single gene biomarker were observed in a few patient cohorts, an EHMT2 signature consisting of EHMT2 and its associated genes revealed significantly better predictive ability for heterogeneous clinical behaviors in patients with BRC (Fig. 5). In addition, when exploring patient subgroups divided by the EHMT2 signature and clinicopathological factors, the high-risk subgroup classified by the EHMT2 signature (i.e., high-EHMT2 cluster) had strong associations with invasive ductal carcinoma, basal, or TNBC subtypes, which are typical characteristics of patients BRC with poor prognoses (Fig. 6). Thus, it is suggested that EHMT2 has a distinct predictive value for the prognosis of BRC and that the EHMT2 signature is compatible with the clinical and molecular features of BRC.

Although many patient cohorts comprising $>1,000$ BRC samples were analyzed in this study and although a prognostic value of EHMT2 and its associated genes was confirmed, there are several limitations to our study. First, recurrence-free survival is an important prognostic factor in BRC, and the KFSYSCC cohort contained data on this statistic; however, we could not estimate an association between the EHMT2 signature and the recurrence-free survival of BRC as too few recurrence events occurred in this cohort. Second, the available clinical and pathological variables varied among the cohorts, which lowered the confidence of the multivariate analysis involving the EHMT2 signature. Third, although the EHMT2 signature showed significant prognostic relevance, an excessive number of genes (1,765 genes) were involved in the signature, making the direct application of this signature to the generation of a diagnostic panel for BRC patient classification impractical. The construction of a diagnostic panel consisting of a small number of genes from the EHMT2 signature and its validation in larger independent clinical cohorts are critically required in future investigations.

In conclusion, in this study, we found that EHMT2 expression was upregulated in BRC tissues and cell lines, which indirectly 
controlled HSPD1 expression and its localization, thus inhibiting cell apoptosis (Fig. 7C). Moreover, in our cohort study, we identified EHMT2 as a novel prognostic marker, implicating its potential as a therapeutic and prognostic marker for BRC treatment. In future studies, we aim to perform ChiP-seq analysis to identify the direct target genes of EHMT2. We will then attempt to clarify the 'mode of action' in HSPD1 regulation in BRC. In addition, we will develop a screening system for the identification of an EHMT2 inhibitor and will perform a pre-clinical study.

\section{Acknowledgements}

Not applicable.

\section{Funding}

This study was supported by a grant from the National Research Foundation of Korea (NRF) grant funded by the Ministry of Science, ICT and Future Planning (NRF-2017R1A2B4003757 and NRF-2018M3A9H3023077), the Technology Innovation Program (No. 10063334) funded by the Ministry of Trade, Industry and Energy (MI, Korea), and the KRIBB Research Initiative Program.

\section{Availability of data and materials}

All data that are not included in this study are available from the corresponding author on reasonable request.

\section{Authors' contributions}

DSK, MYS, HSC, CRJ, RH and SKK were involved in the conception and design of the study. KK and TYR were involved in the development of the methodology. JWR, JHO, MYS, JHL, JKM and SKK were involved in the analysis and interpretation of the data (e.g., statistical analysis, biostatistics, computational analysis). KK, TYR, JHL, CRJ, RH, SKK, DSK, MYS and HSC were involved in the writing, reviewing and/or revision of the manuscript. CRJ was responsible for administrative, technical or material support. DSK, MYS, RH and HSC supervised the study. All authors have read and approved the final manuscript.

\section{Ethics approval and consent to participate}

Not applicable.

\section{Patient consent for publication}

Not applicable.

\section{Competing interests}

The authors declare no conflict of interest.

\section{References}

1. Perou CM, Sørlie T, Eisen MB, van de Rijn M, Jeffrey SS Rees CA, Pollack JR, Ross DT, Johnsen H, Akslen LA, et al: Molecular portraits of human breast tumours. Nature 406: $747-752,2000$
2. Sørlie T, Perou CM, Tibshirani R, Aas T, Geisler S, Johnsen H, Hastie T, Eisen MB, van de Rijn M, Jeffrey SS, et al: Gene expression patterns of breast carcinomas distinguish tumor subclasses with clinical implications. Proc Natl Acad Sci USA 98: 10869-10874, 2001.

3. Pasculli B, Barbano R and Parrella P: Epigenetics of breast cancer: Biology and clinical implication in the era of precision medicine. Semin Cancer Biol 51: 22-35, 2018.

4. Jovanovic J, Rønneberg JA, Tost J and Kristensen V: The epigenetics of breast cancer. Mol Oncol 4: 242-254, 2010.

5. Messier TL, Gordon JA, Boyd JR, Tye CE, Browne G, Stein JL, Lian JB and Stein GS: Histone H3 lysine 4 acetylation and methylation dynamics define breast cancer subtypes. Oncotarget 7: 5094-5109, 2016.

6. Tachibana M, Sugimoto K, Fukushima T and Shinkai Y: Set domain-containing protein, G9a, is a novel lysine-preferring mammalian histone methyltransferase with hyperactivity and specific selectivity to lysines 9 and 27 of histone H3. J Biol Chem 276: 25309-25317, 2001.

7. Kim K, Son MY, Jung CR, Kim DS and Cho HS: EHMT2 is a metastasis regulator in breast cancer. Biochem Biophys Res Commun 496: 758-762, 2018.

8. Wang YF, Zhang J, Su Y, Shen YY, Jiang DX, Hou YY, Geng MY, Ding J and Chen Y: G9a regulates breast cancer growth by modulating iron homeostasis through the repression of ferroxidase hephaestin. Nat Commun 8: 274, 2017.

9. Park SE, Yi HJ, Suh N, Park YY, Koh JY, Jeong SY, Cho DH, Kim CS and Hwang JJ: Inhibition of EHMT2/G9a epigenetically increases the transcription of Beclin-1 via an increase in ROS and activation of NF-kB. Oncotarget 7: 39796-39808, 2016.

10. Lee JY, Lee SH, Heo SH, Kim KS, Kim C, Kim DK, Ko JJ and Park KS: Novel Function of Lysine Methyltransferase G9a in the Regulation of Sox2 Protein Stability. PLoS One 10: e0141118, 2015.

11. Miura S, Maesawa C, Shibazaki M, Yasuhira S, Kasai S, Tsunoda K, Maeda F, Takahashi K, Akasaka T and Masuda T: Immunohistochemistry for histone h3 lysine 9 methyltransferase and demethylase proteins in human melanomas. Am J Dermatopathol 36: 211-216, 2014.

12. Zhong X, Chen X, Guan X, Zhang H, Ma Y, Zhang S, Wang E, Zhang L and Han Y: Overexpression of G9a and MCM7 in oesophageal squamous cell carcinoma is associated with poor prognosis. Histopathology 66: 192-200, 2015.

13. Chandra D, Choy G and Tang DG: Cytosolic accumulation of HSP60 during apoptosis with or without apparent mitochondrial release: Evidence that its pro-apoptotic or pro-survival functions involve differential interactions with caspase-3. J Biol Chem 282: 31289-31301, 2007.

14. Ghosh JC, Dohi T, Kang BH and Altieri DC: Hsp60 regulation of tumor cell apoptosis. J Biol Chem 283: 5188-5194, 2008.

15. Kaigorodova EV and Bogatyuk MV: Heat shock proteins as prognostic markers of cancer. Curr Cancer Drug Targets 14: 713-726, 2014.

16. Lee JH, Cho HS, Lee JJ, Jun SY, Ahn JH, Min JS, Yoon JY, Choi MH, Jeon SJ, Lim JH, et al: Plasma glutamate carboxypeptidase is a negative regulator in liver cancer metastasis. Oncotarget 7: 79774-79786, 2016.

17. Ciriello G, Gatza ML, Beck AH, Wilkerson MD, Rhie SK, Pastore A, Zhang H, McLellan M, Yau C, Kandoth C, et al; TCGA Research Network: Comprehensive Molecular Portraits of Invasive Lobular Breast Cancer. Cell 163: 506-519, 2015.

18. Jung KB, Lee H, Son YS, Lee JH, Cho HS, Lee MO, Oh JH, Lee J, Kim S, Jung CR, et al: In vitro and in vivo imaging and tracking of intestinal organoids from human induced pluripotent stem cells. FASEB J 32: 111-122, 2018.

19. Jung KB, Lee H, Son YS, Lee MO, Kim YD, Oh SJ, Kwon O, Cho S, Cho HS, Kim DS, et al: Interleukin-2 induces the in vitro maturation of human pluripotent stem cell-derived intestinal organoids. Nat Commun 9: 3039, 2018.

20. Kim DS, Ryu JW, Son MY, Oh JH, Chung KS, Lee S, Lee JJ, Ahn JH, Min JS, Ahn J, et al: A liver-specific gene expression panel predicts the differentiation status of in vitro hepatocyte models. Hepatology 66: 1662-1674, 2017.

21. Son MY, Jung CR, Kim DS and Cho HS: Comparative in silico profiling of epigenetic modifiers in human tissues. Mol Biol Rep 45: 309-314, 2018.

22. Ryu JW, Kim SK, Son MY, Jeon SJ, Oh JH, Lim JH, Cho S, Jung CR, Hamamoto R, Kim DS, et al: Novel prognostic marker PRMT1 regulates cell growth via downregulation of CDKN1A in HCC. Oncotarget 8: 115444-115455, 2017. 
23. Liu J, Lichtenberg T, Hoadley KA, Poisson LM, Lazar AJ, Cherniack AD, Kovatich AJ, Benz CC, Levine DA, Lee AV, et al; Cancer Genome Atlas Research Network: An Integrated TCGA Pan-Cancer Clinical Data Resource to Drive High-Quality Survival Outcome Analytics. Cell 173: 400-416.e11, 2018.

24. Arya RK, Singh A, Yadav NK, Cheruvu SH, Hossain Z, Meena S, Maheshwari S, Singh AK, Shahab U, Sharma C, et al: Anti-breast tumor activity of Eclipta extract in-vitro and in-vivo: Novel evidence of endoplasmic reticulum specific localization of Hsp60 during apoptosis. Sci Rep 5: 18457, 2015.

25. Chang Y, Zhang X, Horton JR, Upadhyay AK, Spannhoff A, Liu J, Snyder JP, Bedford MT and Cheng X: Structural basis for G9a-like protein lysine methyltransferase inhibition by BIX-01294. Nat Struct Mol Biol 16: 312-317, 2009.
26. Lebret T, Watson RW, Molinié V, O'Neill A, Gabriel C, Fitzpatrick JM and Botto H: Heat shock proteins HSP27, HSP60, HSP70, and HSP90: Expression in bladder carcinoma. Cancer 98: 970-977, 2003.

27. Cho HS, Shimazu T, Toyokawa G, Daigo Y, Maehara Y, Hayami S, Ito A, Masuda K, Ikawa N, Field HI, et al: Enhanced HSP70 lysine methylation promotes proliferation of cancer cells through activation of Aurora kinase B. Nat Commun 3: 1072, 2012. Attribution-NonCommercial-NoDerivatives 4.0 International (CC BY-NC-ND 4.0) License. 